\title{
COVALENT CROSS-LINKING OF LIVER COLLAGEN BY PYRIDINOLINE INCREASES IN THE COURSE OF EXPERIMENTAL ALVEOLAR ECHINOCOCCOSIS
}

\author{
RICARD-BLUM S.*, LIANCE M.**, HOUIN R.**, GRIMAUD J.A.* \& VUITTON D.A.***
}

\section{Summary :}

We report that covalent cross-linking of collagen molecules by pyridinoline increases significantly in liver in a murine model of alveolar echinococcosis. The highest amount of pyridinoline per collagen molecule (up to 3.5 fold the control values) is found in liver parasitic lesions. It is also increased, but to a far lesser extent, at distance from the fibrotic areas, in macroscopically normal zones of the liver, suggesting that the increase in mature collagen cross-linking occurring in the fibrogenesis due to Echinococcus multilocularis infection involves the whole liver. The comparison of these data with those we have obtained in another parasitic disease, murine schistosomiasis leading to a milder liver fibrosis, largely reversible following chemotherapy, supports a relationship between the liver pyridinoline level and the severity of liver fibrosis. Pyridinoline could be a tissular marker of chronic liver fibrosis in parasitic diseases.

KEY WORDS : alveolar echinococcosis. collagen. cross-linking. Echinococcus multilocularis. liver fibrosis.

\section{INTRODUCTION}

T

iver fibrosis involves complex quantitative and qualitative changes in the extracellular matrix. It is associated with an increase of most of the extracellular matrix macromolecules, with changes in their microcomposition and in their topographic redistribution (Gressner, 1991). We have shown that the increase in covalent collagen cross-linking, taking place predominantly in the extracellular space, is one of the subtle changes occurring in liver fibrosis (Ricard-Blum et al., 1992a). It is initiated by lysyl oxidase which catalyzes the oxidative deamination of $\varepsilon$-amino groups of certain lysine and hydroxylysine residues. The resulting aldehydes condense with

* CNRS URA 1459, Institut Pasteur de Lyon, 69365 Lyon cédex 07, France

** Laboratoire de Parasitologie, Faculté de Médecine, 94010 Créteil Cedex, France

*** Groupe de Recherche sur l'Echinococcose Alvéolaire (AREA), Faculté de Médecine, 25030 Besançon cédex, France.

Corresponding author: Dr Sylvie Ricard-Blum, CNRS URA 1459, Pathologie des Fibroses, Institut Pasteur de Lyon, avenue Tony Garnier, 69365 Lyon cédex 07, France. Fax : (33) 78611345 Tel : (33) 72722514.
Résumé : La RÉTICULATION COVALENTE DU COLLAGÈNE hÉPATIQUE PAR LA PYRIDINOLINE AUGMENTE AU COURS DE L'ÉCHINOCOCCOSE ALVÉOLAIRE MURINE

La réticulation covalente des molécules de collagène par la pyridinoline augmente significativement dans le foie au cours de l'échinococcose alvéolaire provoquée chez la souris par Echinococcus multilocularis. Le taux de pyridinoline, exprimé en nombre de résidus par molécule de collagène, est le plus élevé dans les lésions fibreuses du foie (jusqu'à 3,5 fois supérieur à la valeur normale). La réticulation augmente également, mais dans une moindre mesure, à distance des lésions dans les zones macroscopiquement normales du foie. La fibrogenèse due à l'infection par Echinococcus multilocularis semble donc impliquer la totalité du foie. La comparaison de ces résultats avec ceux que nous avons obtenus dans une autre maladie parasitaire, la bilharziose murine qui provoque une fibrose hépatique plus modérée, indique l'existence d'une relation entre un taux hépatique élevé de pyridinoline et la sévérité de la fibrose hépatique. La pyridinoline pourrait être un marqueur tissulaire de la chronicité des fibroses hépatiques d'origine parasitaire.

MOTS CLES : collagène. échinococcose alvéolaire. Echinococcus multilocularis. fibrose hépatique. réticulation.

$\varepsilon$-amino groups of other lysine and hydroxylysine residues to form intermolecular reducible cross-links which are the biosynthetic precursors of the mature intermolecular cross-links that form spontaneously upon aging (Ricard-Blum and Ville, 1989; Reiser et al., 1992 for reviews).

We have previously found very high hepatic levels of one of these mature cross-links, pyridinoline, in liver biopsies from patients with alveolar echinococcosis (Ricard-Blum et al., 1992a). This uncommon and very severe disease, mostly fatal in humans, is due to the development in the liver of the larval form of the cestode Echinococcus multilocularis. It is associated to an extensive granulomatous reaction surrounding the parasitic vesicles (Vuitton et al., 1989; Bresson-Hadni et al., 1990) and to an intense fibrogenesis leading to a dense and irreversible fibrosis. By contrast, we have observed a moderate increase in liver pyridinoline in the course of another parasitic disease, murine schistosomiasis leading to a largely reversible liver fibrosis (Ricard-Blum et al., 1992b). We have hypothesized from these data that a high level of pyridinoline in liver, and essentially a high number of pyridinoline residues per liver collagen molecule, could be a mar- 
ker of non reversible, chronic, fibrosis. However, the comparison between the reversible and the non reversible fibrosis was impaired by the species difference, since we have studied alveolar echinococcosis in humans and schistosomiasis in a murine experimental model.

The present study reports the measurement of liver pyridinoline in a murine model of alveolar echinococcosis, reproducing with good accuracy and reliability the changes observed in humans (Liance et al., 1984). It was designed 1) to follow the changes occurring in liver pyridinoline in the course of the fibrogenesis induced by E. multilocularis infection, and not only in a single end point like in humans, and 2) to compare the results obtained in this model of non reversible fibrosis with those collected in an experimental model of a largely reversible liver fibrosis, schistosomiasis, in order to precise the relationship between the pyridinoline content of liver collagen molecules and the reversibility of liver fibrosis from parasitic origin.

\section{METHODS}

\section{EXPERIMENTAL MODEL OF ALVEOLAR ECHINOCOCCOSIS}

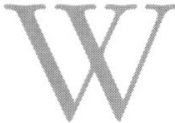

e used a murine model of intra-hepatic Echinococcus multilocularis infection in inbred AKR mice, previously shown to be highly susceptible to this infection, and characterized by a significant primary liver lesion associated with frequent peritoneal metastases (Liance et al., 1984). E. multilocularis strain was obtained from a natural host Arvicola terrestris in Franche-Comté and was subsequently maintained by successive intra-peritoneal injections in Meriones unguiculatus. Intra-hepatic injection of E. multilocularis larvae in the anterior lobe of liver, allowing the study of both the infected and the non-infected lobes in infected mice, was performed after midline laparotomy under anaesthesia (Liance et al., 1984). The modifications due to the injection itself were evaluated in control mice, which received an intra-hepatic injection of saline, using the same surgical procedure. The animals were fed a pelleted diet and tap water at libitum. They were kept under a 12-hr light-dark cycle with constant temperature and humidity in animal care facilities registered by the French Ministry of Agriculture.

Five infected and two control mice were sacrificed at 1, 2, 4 and 6 months post-infection. The non-infected and the infected lobes (infected mice) and the noninjected and the saline-injected lobes (control mice) were stored frozen at $-20^{\circ} \mathrm{C}$. In infected mice, parasitic lesions appeared in the infected lobe as white masses at 4 and 6 months post-infection, and were separated (in the frozen state) from macroscopically normal areas of the same lobe. The normal zones and the fibrotic lesions of the infected lobe were analyzed separately at these times.

\section{PYRIDINOLINE AND HYDROXYPROLINE ANALYSIS}

Liver samples were freeze-dried and hydrolyzed by $6 \mathrm{~N} \mathrm{HCl}$ at $108^{\circ} \mathrm{C}$ for $24 \mathrm{~h}$. One part of the hydrolysates was used for hydroxyproline measurement by a colorimetric method (Woessner, 1961). Collagen content, expressed as g/100 g of liver (dry weight), was calculated by taking into account that collagen contains $13 \%$ of hydroxyproline. The inoculum of $E$. multilocularis larvae used for the infection of mice was found to contain a negligible amount of collagen $(0.46 \%)$ and thus did not significantly contribute to the hepatic pyridinoline level, pyridinoline being found in collagen only.

For pyridinoline analysis, the hydrolysate was first submitted to partition chromatography on CF1 cellulose (Black et al., 1988). Freeze-dried extracts, corresponding to $200-400 \mu \mathrm{g}$ of liver (dry weight), were analyzed by reversed-phase high-performance liquid chromatography as previously described (Ricard-Blum et al., 1992b). Pyridinoline was detected by its natural fluorescence ( $\lambda$ excitation : $295 \mathrm{~nm}, \lambda$ emission : 395 $\mathrm{nm})$. Calibration of the column was performed with purified pyridinoline (generous gift of Dr. S.P. Robins, Rowett Research Institute, Aberdeen, Scotland). Pyridinoline was expressed as pmol/100 $\mu \mathrm{g}$ of liver and as $\mathrm{pmol} / \mathrm{pmol}$ of collagen, assuming a molecular weight of 300,000 for the collagen molecule.

\section{STATISTICAL ANALYSIS}

To compare the data between non-infected and infected lobe and between parasitic lesions and normal areas in infected lobe, we used Student's t test with significance set at $\mathrm{p}<0.05$.

\section{RESULTS}

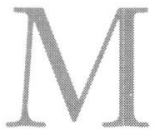
ice responded similarly to E. multilocularis infection within each group, except at 2 months post-infection. At this time, one mouse failed to develop apparent parasitic lesions and was excluded from the study. Three mice became infected and the parasitic lesions were too small, as observed also at 1 month post-infection, to allow their separation from the normal areas of the infected lobe. Only one mouse developed extensive parasitic lesions, clearly distinct from the surrounding 
parenchyma. These two zones of the infected lobe were analyzed separately for collagen and pyridinoline, as performed for all infected mice at 4 and 6 months post-infection. However the values obtained for this particular mouse were not included in the calculation of the mean collagen and pyridinoline contents, as only a single value resulting from the analysis of the whole liver was obtained for the other mice sacrificed at this time.

In the liver, mean collagen content remained below $1 \%(\mathrm{~g} / 100 \mathrm{~g}$ of liver $)$ in both sham-injected and noninjected lobes of control mice and also in the noninfected lobe of infected mice during the course of the experiment (Fig. 1). By contrast, collagen increased progressively in the infected lobe, up to $8.3 \%$ (mean value) in parasitic lesions at 4 months postinfection. There was no further increase in total liver collagen content between 4 and 6 months post-infection (Fig. 1). At 4 and 6 months post-infection, collagen content was significantly higher in fibrotic areas than in macroscopically normal zones of the infectedlobe $(p=0.005$ and 0.003 at 4 and 6 months after infection) and than in non-infected lobe $(p=0.0025$ and 0.0014 respectively). Collagen level from macroscopically normal zones of the infected lobe was slightly, but not significantly, higher than in control livers and than in the non-infected lobe of infected mice, except at 6 months post-infection where the difference with the non-infected lobe reached significance $(\mathrm{p}=0.04)$.

The amount of pyridinoline in liver increased slightly throughout the experiment in control mice and was higher in the saline-injected lobe than in the noninjected one (Fig. 2). A similar increase was observed in the non-infected lobe of infected mice. Pyridinoline content of the infected lobe was higher than in controls and than in the non-infected lobe and reached the highest values in parasitic lesions (12.4 and $18.6 \mathrm{pmol} / 100 \mu \mathrm{g}$ of liver at 4 and 6 months post-infection respectively) (Fig. 2). A very pronounced increase occurred in the fibrotic zones between 2 and 6 months, whereas pyridinoline level did not vary significantly in the normal areas of the infected lobe between 2 and 6 months after infection (Fig. 2). The pyridinoline level in fibrotic lesions was significantly higher than in normal zones of the infected lobe ( $p=0.009$ and 0.004 at 4 and 6 months postinfection) and than in the non-infected lobe $(\mathrm{p}=$ 0.005 and 0.003 at 4 and 6 months).

The mean number of pyridinoline residues per collagen molecule increased slightly in the course of the disease in saline-injected lobe of control mice, and increased to a higher extent in infected mice (Fig. 3). It was higher in the liver of infected mice (either infected or non-infected lobe) than in controls. The number of

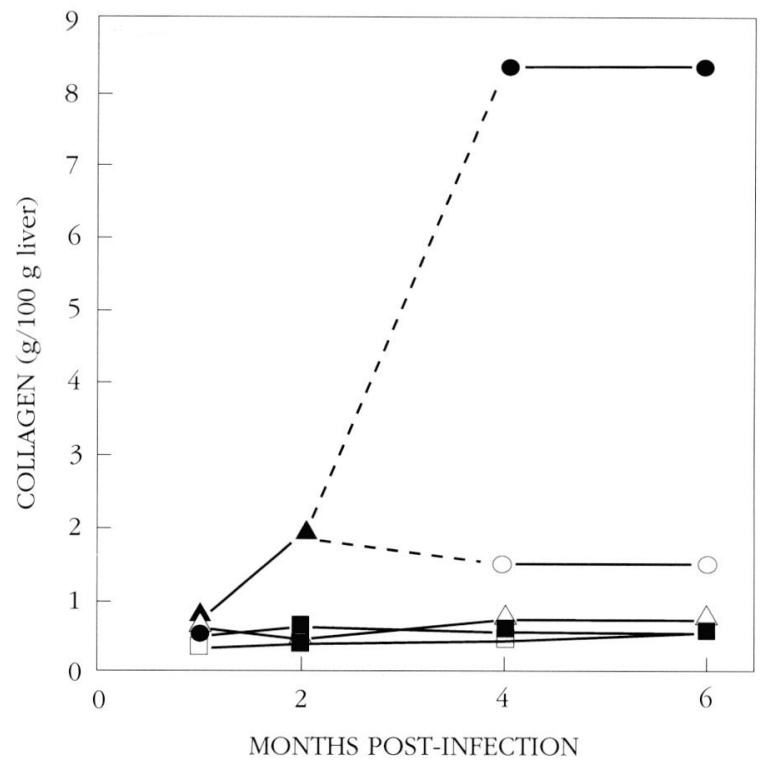

Fig. 1 : Total liver collagen content ( $\mathrm{g} / 100 \mathrm{~g}$ of liver, dry weight) in the course of murine alveolar echinococcosis. Control mice (n $=2)$ : non-injected $(\square)$ and saline-injected ( $\square$ ) lobes. Infected mice ( $\mathrm{n}=5$, except at 2 months where $\mathrm{n}=3$ ) : non-infected $(\triangle)$ and infected lobe $(\boldsymbol{\Lambda})$ that was divided into macroscopically normal liver $(O)$ and parasitic lesions $(\bullet)$ at 4 and 6 months post-infection.

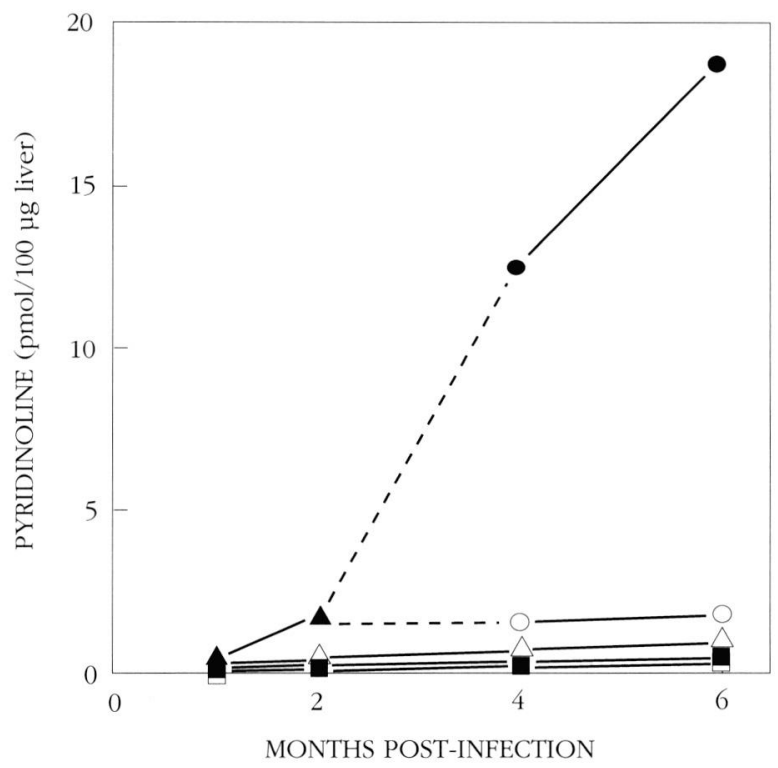

Fig. 2 : Hepatic pyridinoline level (expressed as pmol/100 $\mu \mathrm{g}$ of liver, dry weight) in the course of murine alveolar echinococcosis. Control mice $(\mathrm{n}=2)$ : non-injected $(\square)$ and saline-injected ( $\square$ ) lobes. Infected mice ( $n=5$, except at 2 months where $n=3)$ : non-infected $(\triangle)$ and infected lobe $(\boldsymbol{\Delta})$ that was divided into macroscopically normal liver $(O)$ and parasitic lesions $(\bullet)$ at 4 and 6 months post-infection. 


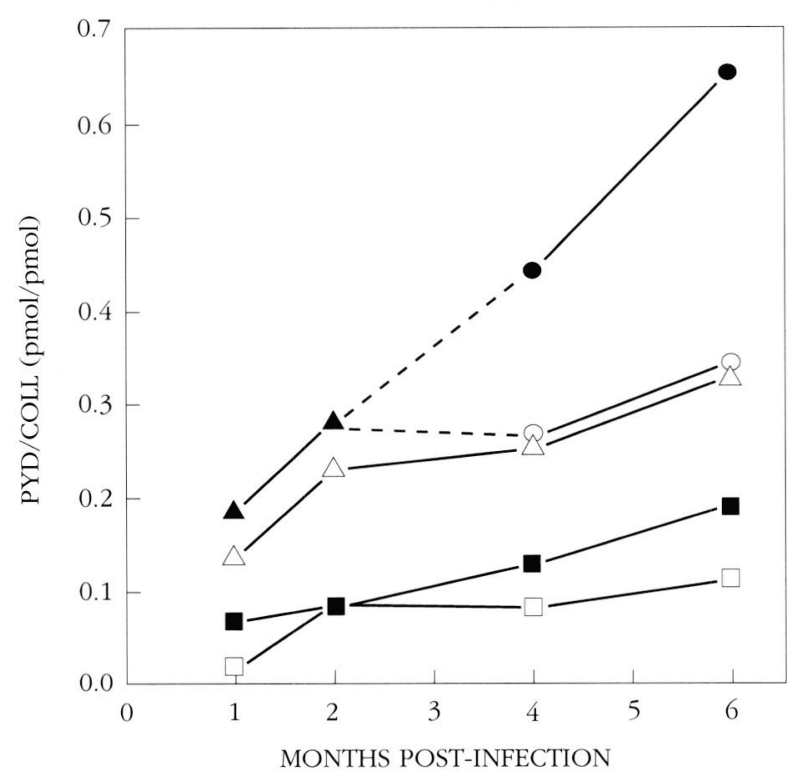

pyridinoline cross-links per collagen molecule markedly increased in the fibrotic lesions of the infected lobe between 4 and 6 months post-infection (Fig. 3), and was significantly higher than in normal zones of the infected lobe $(p=0.007$ and $p=0.001$ at 4 and 6 months) and than in the non-infected lobe ( $p=0.004$ and $\mathrm{p}=0.0001$ at 4 and 6 months). The pyridinoline/collagen ratio was similar in the normal part of the infected lobe and in the non-infected lobe between 4 and 6 months after infection.

\section{DISCUSSION}

F irst, this study demonstrates that pyridinoline significantly increases in liver, both at the tissue and collagen levels, in the course of the non reversible fibrogenesis induced by E. multilocularis infection. A slight increase in the collagen crosslinking by pyridinoline was found throughout the experiment in control livers and this likely reflects the physiological formation of pyridinoline which occurs through normal aging. Sham-injection with saline was also followed by a slight and transient increase in the collagen level and in the number of pyridinoline residues per collagen molecule. This could be due to the discrete inflammatory reaction following the injection, a phenomenon we have previously observed in this model using another technique for collagen measurement, namely a micromethod in paraffin-embedded sections (Vuitton et al., 1986a). In infected mice, the most pronounced changes were observed in fibrotic lesions, whereas cross-linking by pyridinoline in normal areas of infected lobe and in non-infected lobe was moderately increased when compared to
Fig. 3 : Number of pyridinoline residues per liver collagen molecule, expressed as $\mathrm{pmol} / \mathrm{pmol}$ of collagen, in the course of murine alveolar echinococcosis. Control mice $(\mathrm{n}=2)$ : non-injected $(\square)$ and salineinjected ( $\mathbf{a})$ lobes. Infected mice $(n=5$, except at 2 months where $\mathrm{n}=3)$ : non-infected $(\Delta)$ and infected lobe $(\mathbf{\Delta})$ that was divided into macroscopically normal liver (O) and parasitic lesions (•) at 4 and 6 months post-infection. controls. These results support the involvement of collagen molecules from the whole liver in the course of fibrogenesis. Indeed, a thickening of the normal collagen framework has been observed in the liver parenchyma distant from the fibrotic zones in both experimental and human alveolar echinococcosis (Vuitton et al., 1986a, b). The maturation of collagen cross-linking, reflected by pyridinoline formation, occurs to a higher rate and to a higher extent in infected livers, specially in fibrotic lesions, than in age-matched controls. This suggests that fibrogenesis induced by Echinococcus multilocularis infection could accelerate the maturation of collagen cross-linking, an age-related process.

The involvement of the whole liver is probably mediated by cytokines. In regard to collagen crosslinking, the only enzymatic step identified so far is the first one, catalyzed by lysyl oxidase. The following reactions, leading to reducible and mature cross-links, are believed to be spontaneous and it seems unlikely that cytokines could directly affect them, although an increase in lysyl oxidase expression and/or activity mediated by cytokines might contribute to the increase in cross-linking. Fibrogenic cytokines (Kovacs, 1991), such as TGF-ß that interferes with proteolytic degradation of the extracellular matrix by reducing synthesis and secretion of several proteases and by increasing synthesis of specific inhibitors (Roberts et al., 1992), could indirectly contribute to the observed increase in pyridinoline. Indeed, the impairment of collagen degradation could let more time for the completion of cross-linking maturation in the collagen molecules deposited in the extracellular matrix. A perpetuating factor contributing to the slowing-down of collagen turn-over could be the 
increase in pyridinoline residues per collagen molecule itself, as it begins early in fibrogenesis and is capable to induce a resistance within collagen molecules to degradation by collagenase (Vater et al., 1979). Data on matrix metalloproteinases in the course of alveolar echinoccocosis would be helpful to better understand the mechanism of mature cross-linking increase in this model.

Since AKR mice exhibited an extensive fibrosis with late and definitive deposition of type I collagen, the most probable target for pyridinoline formation is type I collagen which forms the bulk of fibrotic collagen and surrounds old parasitic vesicles (Vuitton et al., 1986a). Type III collagen, surrounding active parasitic vesicles, is likely involved to a lesser extent since its deposition is an early event whereas the number of pyridinoline residues per collagen molecule reaches its highest value at 6 months post-infection. Among the other collagen types so far identified in fibrotic liver, type $\mathrm{V}$ collagen, but neither type IV nor type VI that are devoided of pyridinoline (Reiser et al., 1992), could also participate in mature cross-linking.

In this study, infected mice exhibited parasitic lesions to a variable extent at 2 months post-infection. At this time, one out of the four analyzed mice developed large fibrotic lesions, usually encountered later in the experimental infection ( 4 and 6 months). These lesions contained a higher concentration of pyridinoline than the infected lobe of the three other mice. Individual variations in the hepatic parasite burden could contribute to the heterogeneity in the size of fibrotic lesions observed at this time (Liance et al., 1992). However, this phenomenon might also be related to the disease itself, which is a unique example of zonal hepatic fibrosis with very localized parasitic areas and could account for this heterogeneity.

The results obtained in this model of irreversible fibrosis can be compared to those we have previously obtained in a murine model of reversible fibrosis, schistosomiasis, which is partially reversible due to the spontaneous modulation of egg-induced granuloma and considered as largely reversible after anti-parasitic treatment. The highest values of liver pyridinoline were found in alveolar echinococcosis where they reached $18.5 \mathrm{pmol} / 100 \mu \mathrm{g}$ of liver compared to $7.2 \mathrm{pmol} / 100 \mu \mathrm{g}$ of liver in schistosomiasis. The number of pyridinoline residues per collagen molecule was also higher in experimental alveolar echinococcosis (mean value : $0.65 \mathrm{pmol} / \mathrm{pmol}$ of collagen in fibrotic lesions at 6 months post-infection) than in experimental schistosomiasis $(0.47 \mathrm{pmol} / \mathrm{pmol}$ of collagen at 9 months post-infection, Ricard-Blum et al., 1992b). We have used AKR mice for echinococcosis and Balb/c mice for schistosomiasis and this might contribute to the difference observed between absolute levels of cross-linking, thus we have also considered the relative increase in pyridinoline compared to the controls. Liver pyridinoline increased up to 12 -fold in schistosomiasis and up to 59-fold in alveolar echinococcosis. The number of pyridinoline residues per collagen molecule increased up to two fold above the control values in schistosomiasis, whereas in alveolar echinococcosis a 3.5-fold increase was observed in the fibrotic lesions compared to the saline-injected controls. The comparison between these two models of liver fibrosis differing by their degree of reversibility shows that pyridinoline content is higher in the non reversible fibrosis, both at the liver and collagen levels. This supports our hypothesis that a marked increase in the number of pyridinoline residues per collagen molecule, corresponding to an irreversible modification of the stability of the collagen network, is related to the severity of liver fibrosis. Pyridinoline measurement could therefore be proposed as a reliable tissular marker of the degree of severity of liver fibrosis. Requirement for tissue samples could limit the clinical use of this parameter, but a promising perspective for clinical application is the fact that pyridinoline is excreted in urine (GunjaSmith and Boucek, 1981). This would make possible the follow-up of pyridinoline in liver fibrogenesis by a non invasive method. Further studies are currently in progress in the laboratory to determine if urinary and hepatic levels of pyridinoline are correlated in the course of experimental liver fibrosis.

\section{ACKNOWLEDGEMENTS}

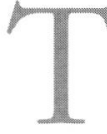
he authors gratefully acknowledge the expert technical assistance from B. Blanquier, C. Juillet and D. Rivollet and the generous gift of purified pyridinoline by Dr. S.P. Robins (Rowett Research Institute, Aberdeen, Scotland). This work was supported by a grant from the Direction de la Recherche et des Etudes Doctorales du Ministère de l'Éducation nationale.

\section{REFERENCES}

Black D., Duncan A. \& Robins S.P. Quantitative analysis of the pyridinium cross-links of collagen in urine using ionpaired reversed-phase high-performance liquid chromatography. Analytical Biochemistry, 1988, 169, 197-203.

Bresson-Hadni S., Liance M., Meyer J.P., Houin R., Bresson J.L. \& VuitTon D.A. Cellular immunity in experimental Echinococcus multilocularis infection. 2) Sequential and comparative phenotypic study of the periparasitic mononuclear cells in resistant and sensitive mice. Clinical and Experimental Immunology, 1990, 82, 378-83. 
Gressner A.M. Liver fibrosis : Perspectives in pathobiochemical research and clinical outlook. European Journal of Clinical Chemistry and Clinical Biochemistry, 1991, 29, 293-311.

Gunja-Smith Z. \& Boucek R.J. Collagen cross-linking compounds in human urine. Biochemical Journal, 1981, 197, 759-762.

Kovacs E.J. Fibrogenic cytokines : the role of immune mediators in the development of scar tissue. Immunology Today, 1991, 12, 17-23.

Liance M., Vuitton D., Guerret-Stocker S., Carbillet J.P., Grimaud J.A. \& Houin R. Experimental alveolar echinococcosis. Suitability of a murine model of intra-hepatic infection by E. multilocularis for immunological studies. Experientia, 1984, 40, 1436-1439.

Liance M., Bresson-Hadni S., Vuitton D.A., Lenys D., CarBILlet J.P. \& Houin R. Effects of cyclosporin A on the course of murine alveolar echinococcosis and on specific cellular and humoral immune responses against Echinococcus multilocularis. International Journal of Parasitology, 1992, 22, 23-28.

Reiser K., McCormick R.J. \& Rucker R.B. Enzymatic and nonenzymatic cross-linking of collagen and elastin. FASEB Journal, 1992, 6, 2439-2449.

Ricard-Blum S. \& Ville G. Collagen cross-linking. International Journal of Biochemistry, 1989, 21, 1185-1189.

Ricard-Blum S., Bresson-Hadni S., Vuttton D.A., Ville G. \& Grimaud J.A. Hydroxypyridinium collagen cross-links in human liver fibrosis : study of alveolar echinococcosis. Hepatology, 1992a, 15, 599-602.

Ricard-Blum S., Ville G. and Grimaud J.A. Pyridinoline, a mature collagen cross-link, in fibrotic livers from Schistosoma mansoni-infected mice. American Journal of Tropical Medicine and Hygiene, 1992b, 47, 816-820.

Roberts A.B., McCune B.K. and Sporn M.B. TGF-ß : regulation of extracellular matrix. Kidney International, 1992, 41, 557-559.

VAter C.A., Harris E.D. \& Siegel R.C. Native cross-links in collagen fibrils induce resistance to human synovial collagenase. Biochemical Journal, 1979, 181, 639-645.

Vuitton D.A., Tran V.P., Guerret-Stocker S., Grimaud J.A., Liance M. \& Houin R. Development of fibrosis in hepatic alveolar echinococcosis : a sequential study in mice infected with Echinococcus multilocularis. In : Bienvenu J, Grimaud JA, Laurent P, eds. Marker Proteins inflammation, vol 3. Berlin : Walter de Gruyter \& Co, 1986a ; 491-500.

Vuitton D.A., Guerret-Stocker S., Carbillet J.P., Mantion G., Miguet J.P. \& GRimaud J.A. Collagen immunotyping of the hepatic fibrosis in human alveolar echinococcosis. Zeitschrift für Parasitenkunde (Parasitology Research), 1986b, 72, 97-104.

Vuitton D.A., Bresson-Hadni S., Laroche L., Kaiserlian D., Guerret-Stocker S., Bresson J.L. \& Gillet M. Cellular immune response in Echinococcus multilocularis infection in humans. 2) Natural killer cell activity and cell subpopulations in the blood and in the periparasitic granuloma of patients with alveolar echinococcosis. Clinical and Experimental Immunology, 1989, 78, 67-74.
WoEsSNER J.F. The determination of hydroxyproline in tissue and protein samples containing small proportions of this imino-acid. Archives of Biochemistry and Biophysics, 1961, 93, 440-47.

Accepté le 26 janvier 1995 